NASA/CR-1998-206921

ICASE Report No. 98-12

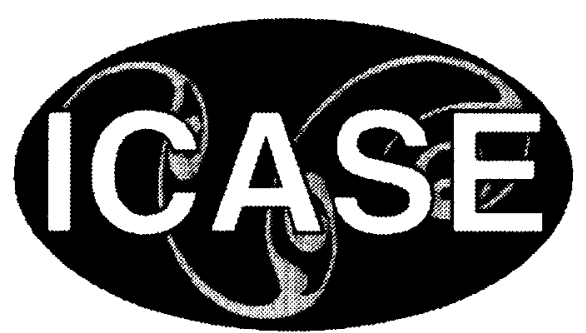

\title{
A Stable and Conservative Interface Treatment of Arbitrary Spatial Accuracy
}

Mark H. Carpenter, Jan Nordstrom, and David Gottlieb 


\section{The NASA STI Program Office ... in Profile}

Since its founding, NASA has been dedicated to the advancement of aeronautics and space science. The NASA Scientific and Technical Information (STI) Program Office plays a key part in helping NASA maintain this important role.

The NASA STI Program Office is operated by Langley Research Center, the lead center for NASA's scientific and technical information. The NASA STI Program Office provides access to the NASA STI Database, the largest collection of aeronautical and space science STI in the world. The Program Office is also NASA's institutional mechanism for disseminating the results of its research and development activities. These results are published by NASA in the NASA STI Report Series, which includes the following report types:

- TECHNICAL PUBLICATION. Reports of completed research or a major significant phase of research that present the results of NASA programs and include extensive data or theoretical analysis. Includes compilations of significant scientific and technical data and information deemed to be of continuing reference value. NASA counter-part or peer-reviewed formal professional papers, but having less stringent limitations on manuscript length and extent of graphic presentations.

- TECHNICAL MEMORANDUM. Scientific and technical findings that are preliminary or of specialized interest, e.g., quick release reports, working papers, and bibliographies that contain minimal annotation. Does not contain extensive analysis.

- CONTRACTOR REPORT. Scientific and technical findings by NASA-sponsored contractors and grantees.
- CONFERENCE PUBLICATIONS. Collected papers from scientific and technical conferences, symposia, seminars, or other meetings sponsored or co-sponsored by NASA.

- SPECIAL PUBLICATION. Scientific, technical, or historical information from NASA programs, projects, and missions, often concerned with subjects having substantial public interest.

- TECHNICAL TRANSLATION. Englishlanguage translations of foreign scientific and technical material pertinent to NASA's mission.

Specialized services that help round out the STI Program Office's diverse offerings include creating custom thesauri, building customized databases, organizing and publishing research results ... even providing videos.

For more information about the NASA STI Program Office, you can:

- Access the NASA STI Program Home Page at http://www.sti.nasa.gov/STIhomepage.html

- Email your question via the Internet to help@sti.nasa.gov

- Fax your question to the NASA Access Help Desk at (301) 621-0134

- Phone the NASA Access Help Desk at (301) 621-0390

- Write to: NASA Access Help Desk NASA Center for AeroSpace Information 800 Elkridge Landing Road Linthicum Heights, MD 21090-2934 
NASA/CR-1998-206921

ICASE Report No. 98-12

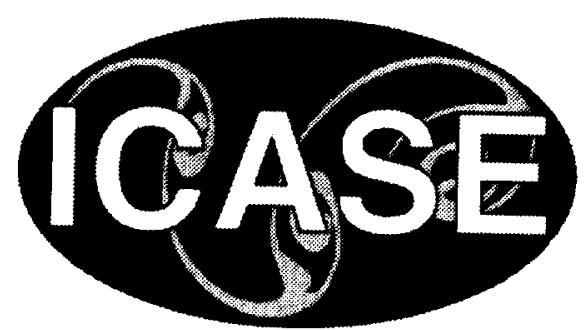

\section{A Stable and Conservative Interface Treatment of Arbitrary Spatial Accuracy}

Mark H. Carpenter

NASA Langley Research Center

Jan Nordstrom

The Aeronautical Research Institute of Sweden

David Gottlieb

Brown University

Institute for Computer Applications in Science and Engineering NASA Langley Research Center

Hampton, VA

Operated by Universities Space Research Association

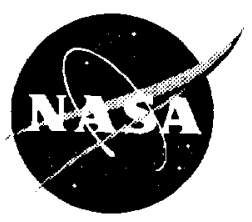

National Aeronautics and

Space Administration

Langley Research Center

Hampton, Virginia 23681-2199

February 1998 
Available from the following:

NASA Center for AeroSpace Information (CASI)

800 Elkridge Landing Road

Linthicum Heights, MD 21090-2934

(301) $621-0390$
National Technical Information Service (NTIS) 5285 Port Royal Road

Springfield, VA 22161-2171

(703) $487-4650$ 


\title{
A STABLE AND CONSERVATIVE INTERFACE TREATMENT OF ARBITRARY SPATIAL ACCURACY*
}

\author{
MARK H. CARPENTER ${ }^{\dagger}$, JAN NORDSTRÖM ${ }^{\ddagger}$, AND DAVID GOTTLIEB ${ }^{\S}$
}

\begin{abstract}
Stable and accurate interface conditions are derived for the linear advection-diffusion equation. The conditions are functionally independent of the spatial order of accuracy and rely only on the form of the discrete operator. We focus on high-order finite-difference operators that satisfy the summation-by-parts (SBP) property. We prove that stability is a natural consequence of the SBP opcrators used in conjunction with the new boundary conditions. In addition, we show that the interface treatments are conservative.

New finite-difference operators of spatial accuracy up to sixth order are constructed: these operators satisfy the SBP property. Finite-difference operators are shown to admit design accuracy ( $p^{\text {th }}$-order global accuracy) when $(p-1)^{t h}$-order stencil closures are used near the boundaries if the physical boundary conditions are implemented to at least $p^{\text {th }}$-order accuracy. Stability and accuracy are demonstrated on the nonlinear Burgers' equation for an twelve-subdomain problem with randomly distributed interfaces.
\end{abstract}

Key words. high-order finite-difference, numerical stability, interface conditions, summation-by-parts

Subject classification. Applied and Numerical Mathematics

1. Introduction. Higher order and spectral schemes are idcally suited for resolving problems for which high resolution is essential. Computational acroacoustics (CAA) and computational electromagnetics (CEM) are two such fields that require high accuracy to resolve the vastly disparate length and time scales involved. High-order (spectral) schemes easily outperform low-order schemes on simple problems in which the physical domain is smoothly mapped onto the computational space. The spatial convergence rates of these schemes allow satisfactory results on relatively coarse grids.

At least two fundamental obstacles presently limit the use of high-order schemes. The first one is the lack of nonlinear robustness exhibited by high-order formulations. Under resolved features in the solution and inappropriate numerical and physical boundary conditions are the primary causes. A second limitation is the difficulty in applying high-order formulations to complex geometries. Often, the gencration of a grid around a complex configuration is the most difficult aspect of the solution procedure. Further constraint of the grids so that they are smooth to higher order (necessary to attain design accuracy for high-order methods) severely complicates grid generation around complex configurations.

Many high-order practitioners advocate a fully unstructured approach to grid generation. This approach simplifies the grid-generation procedure considerably for complex configurations. Finite-clement techniques are an example of the fully unstructured schemes that are routinely used on complex geometries. An alternative to fully unstructured methods is the semistructured approach, in which the solution domain

*This research was supported in part by the National Aeronautics and Space Administration under NASA Contract Nos. NAS1-19480 and NAS1-97046 while the second and third authors were in residence at the Institute for Computer Applications in Science and Engineering (ICASE), NASA Langley Research Center, Hampton, VA 23681-2199.

${ }^{\dagger}$ Aerodynamic and Acoustic Methods Branch, NASA Langley Research Center, Hampton, VA 23681-2199. E-mail: m.h.carpenter@larc.nasa.gov.

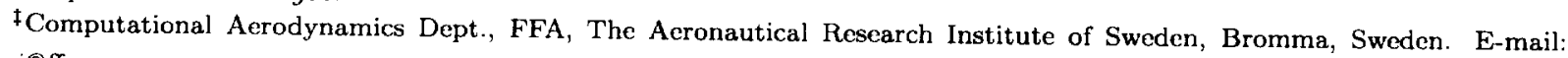
$n m j @ f f a . s e$.

${ }^{\S}$ Division of Applied Mathematics, Brown University, Providence, RI 02912. Work is supported by AFOSR grant F4962096-1-0150 and by NSF grant DMS-9500814. E-mail: dig@cfm.brown.edu. 
is broken into the union of piecewise smooth subdomains. Each subdomain is discretized with a stable formulation, and the resulting multiple domains are patched together globally. This technique has been successfully used by Kopriva [1], and more recently by Hesthaven and Gottlieb [2].

The approach for designing interface conditions developed in this work is equally valid for the unstructured and semi-structured approaches in multiple spatial dimensions. The interface conditions are determined entirely by accurate left and right state data along the interface, and do not depend on the source of the data. For simplicity, however, we focus on the interface matching conditions necessary to maintain stability and accuracy in one spatial dimension. We demonstrate the technique for both spectral and high-order formulations.

In section 2, we define and describe semidiscrete operators that satisfy the SBP convention. In section 3, we introduce new interface boundary conditions for multiple domains. In section 4 , we show that the new conditions are conservative across interfaces. In section 5 , we consider specific examples of the stability and accuracy of finite-difference schemes. In section 6 , we present the conclusions. Finally, in the appendix wc present the stencils used for fourth- and sixth-order finite-difference schemes.

2. Spatial Discretizations. The stable interface conditions presented in this work are valid for spatial discretizations of arbitrary accuracy. To achieve this generality, the spatial discretizations must be of a specific form. Fortunately, most numerical schemes can be put into the required form with only minor modifications. To be more precise we consider discrete spatial derivative operators with the following properties:

\subsection{First-derivative properties.}

1. The first derivative operator defining the numerical derivative $\mathbf{u}_{\mathbf{x}}=\left[\left(\frac{\partial u}{\partial x}\right)_{0}, \ldots,\left(\frac{\partial u}{\partial x}\right)_{N}\right]^{T}$ is

$$
\begin{aligned}
& P \mathbf{u}_{\mathbf{x}}-Q \mathbf{u}=0 \\
& P \mathbf{v}_{\mathbf{x}}-Q \mathbf{v}=P \mathbf{T}_{\mathbf{e}},
\end{aligned}
$$

where $\mathbf{u}=\left[u_{0}(t), u_{1}(t), \ldots, u_{N}(t)\right]^{T}, \mathbf{v}=\left[v\left(x_{0}, t\right), \ldots, v\left(x_{N}, t\right)\right]^{T}$ and $\mathbf{v}_{\mathbf{x}}=\left[\left(\frac{\partial v}{\partial x}\right)_{0}, \ldots,\left(\frac{\partial v}{\partial x}\right)_{N}\right]^{T}$. (The vector $\mathbf{v}$ is the exact solution.) The truncation error $\mathbf{T}_{\mathbf{e}}$ satisfies $\left|\mathbf{T}_{\mathbf{e}}\right|=O(\Delta x)^{m}$ where the quantity $\Delta x$ is defined as the maximum distance between any two neighboring grid points.

2. The matrix $P$ is symmetric and positive definite $(\Delta x) p I \leq P \leq(\Delta x) q I$ where $p$ and $q$ are independent of $N$ with $p>0$ and $q>0$.

3. The matrix $Q$ is nearly skew symmetric and satisfies the property $Q+Q^{T}=D$, where the diagonal matrix $D$ has the form $d_{i, i}=[-1,0, \ldots, 0,1]$ for $i=0,1, \ldots N$. Furthermore, $Q_{0,0}=-\frac{1}{2}$ and $Q_{N, N}=\frac{1}{2}$.

A spatial operator in the form of equation (2.1), which satisfies properties 1 through 3 , is referred to as an SBP operator [3]. All SBP operators automatically lead to an energy estimate for periodic solutions to the linear advection-diffusion equation. In the finite-domain case, an energy estimate exists when an SBP operator is combined with specific boundary treatments.

Discretization operators that satisfy the SBP framework are remarkably general. Kreiss and Scherer [3] first suggested the use of SBP spatial operators in the context of second-order central-difference schemes. In Olsson [4][5][6] and Strand [7], high order finite difference operators are constructed based on spatial operators of SBP type. These resulting schemes are strictly stable which means that the growth rate of the analytic and semi-discrete solution is identical.

The precise properties of the matrices $P$ and $Q$ provide a constructive means of formulating boundary closures. A discretization begins with a parametcrization of several points near the boundary of the required 
accuracy. The parameters are then adjusted so that they match the precise requirements of the $P$ and $Q$ matrices. Strand [8] used the SBP approach to construct stable fourth- and sixth-order central-differencing schemes with boundary closures of the appropriate order. Carpenter, Gottlieb, and Abarbancl [9] extended the SBP formalism to compact implicit operators (fourth-order Pade' operators); Carpenter and Gottlieb [10] showed that spectral formulations (Galerkin and collocation) can be cased in the SBP framework. Finally, Carpenter and Otto [11] showed that the SBP schemes have a natural interface property, and they used this property to derive a class of multiple-domain schemes referred to as "cyclo-difference" schemes. (The earlier work [11] required strong imposition of interface data, whereas the present formulation requires only weak imposition).

The SBP schemes naturally arise with centered approximations, for which the spatial operator is skew symmetric. A more general class of schemes could be formulated in the form

$$
\frac{d \mathbf{u}}{d x}=P^{-1}(Q+T) \mathbf{u}
$$

where the matrix $T$ is symmetric negative definite. The general formulation includes the entire class of central and upwind schemes. The upwind schemes are automatically stable and accurate because they are obtained by adding a symmetric high-order diffusion operator to a stable and accurate SBP formulation. Wc focus, therefore, on the original SBP definition which includes central, compact, and spectral formulations.

An approach similar to that used on the first-derivative operator can be used for the second-derivative operator. For example, one can seek two positive-definite matrices $L$ and $R$ such that

$$
\mathbf{v}_{x x}-L^{-1} R \mathbf{v}=O(\Delta x)^{m}
$$

An obvious choice is to take $L=P$ and $R=Q P^{-1} Q$ so that the second-derivative operator is obtained by repeated differentiation with the first-derivative operator. For spectral discretizations, this process of differentiation is a natural consequence of the polynomial-based discretization technique. This same assumption for finite-difference techniques is acceptable but less desirable than other, more compact formulations. A second derivative formed from two first-derivative operators is unnecessarily wide and inaccurate and can lead to odd-even mode decoupling. For this reason, we seek a second-derivative operator with the following properties:

\subsection{Second-derivative properties.}

1. The second-derivative operator that defines $\mathbf{u}_{x x}$ is

$$
\begin{aligned}
& P \mathbf{u}_{\mathbf{x x}}-\left(-S^{T} M+D\right) S \mathbf{u}=0 \\
& P \mathbf{v}_{\mathbf{x x}}-\left(-S^{T} M+D\right) S \mathbf{v}=P \mathbf{T}_{\mathbf{e} \mathbf{2}}
\end{aligned}
$$

where the diagonal matrix $D$ has the form $d_{i, i}=[-1,0, \ldots, 0,1], i=0,1, \ldots N$

2. The matrix $M$ is positive definite: $(\Delta x) m I \leq M \leq(\Delta x) n I$, where $m$ and $n$ are independent of $N$ with $m>0$ and $n>0$.

3. The matrix $S$ is of the form

$$
S=\frac{1}{(\Delta x)}\left[\begin{array}{cccccccc}
s_{0,0} & s_{0,1} & s_{0,2} & s_{0,3} & \ldots & & \\
0 & 1 & 0 & & & & \\
& 0 & 1 & 0 & & & \\
& & & . & & & \\
& & & 0 & 1 & 0 & \\
& & & & 0 & 1 & 0 \\
& & \ldots & s_{N, N-3} & s_{N, N-2} & s_{N, N-1} & s_{N, N}
\end{array}\right]
$$


where

$$
\begin{gathered}
\left.S u\right|_{0}=v_{x}\left(x_{0}\right)+O(\Delta x)^{r} \\
\left.S u\right|_{N}=v_{x}\left(x_{N}\right)+O(\Delta x)^{r}
\end{gathered}
$$

The matrix $S$ is the identity matrix (scaled by the grid spacing) where a discrete representation of the first derivative replaces the first and last rows.

4. The matrix $P$ is that used in the first-derivative operator.

Explicit forms of the matrices $S$ and $M$ are given in the appendix for a second-order explicit discretization. In addition, the matrix $S$ is presented up to sixth order.

3. Interface Boundary Conditions for Multiple Domains. Consider the linear advection-diffusion equation

$$
\frac{\partial U}{\partial t}+a \frac{\partial U}{\partial x}=\epsilon \frac{\partial^{2} U}{\partial x^{2}}, \quad|x| \leq 1, \quad t>0 .
$$

Suppose that the equation is discretized by a multi-domain technique such that the interval is divided arbitrarily into two subintervals $-1 \leq x \leq x_{i}$ and $x_{i} \leq x \leq 1$. On each subinterval, a discretization is used that satisfies the SBP properties 1 through 3 . We propose implementing the interface boundary conditions by using a penalty treatment of the form

$$
\begin{aligned}
P_{l} \mathbf{u}_{t}+a Q_{l} \mathbf{u} & =\epsilon R_{l} \mathbf{u}+\sigma_{1} \mathbf{e}_{\mathbf{l} i}\left(\left.u\right|_{x=x_{i}}-\left.v\right|_{x=x_{i}}\right)+\sigma_{2} \epsilon \mathbf{e}_{l_{i}}\left[\left.\left(D_{l} \mathbf{u}\right)\right|_{x=x_{i}}-\left.\left(D_{r} \mathbf{v}\right)\right|_{x=x_{i}}\right] \\
P_{r} \mathbf{v}_{t}+a Q_{r} \mathbf{v} & =\epsilon R_{r} \mathbf{v}+\sigma_{3} \mathbf{e}_{\mathbf{r} i}\left(\left.v\right|_{x=x_{i}}-\left.u\right|_{x=x_{i}}\right)+\sigma_{4} \epsilon \mathbf{e}_{\mathbf{r} i}\left[\left.\left(D_{r} \mathbf{v}\right)\right|_{x=x_{i}}-\left.\left(D_{l} \mathbf{u}\right)\right|_{x=x_{i}}\right]
\end{aligned}
$$

where $\mathbf{u}$ is a vector of length $M \mathbf{u}=\left[u_{0}(t), u_{1}(t), \ldots, u_{M}(t)\right]^{T}$ defined in the left domain at the points $\left.\mathbf{x}_{\mathbf{L}}=\left[x_{0}=-1, x_{1}, \ldots, x_{M}=x_{i}\right)\right]^{T}$ and $\mathbf{e}_{1_{i}}=[0, \ldots, 0,1]^{T}$ is of dimension $M$. In the right domain, $\mathbf{v}=$ $\left[v_{0}(t), v_{1}(t), \ldots, v_{N}(t)\right]^{T}$ is defined at the points $\mathbf{x}_{\mathbf{R}}=\left[x_{0}=x_{i}, x_{1}, \ldots, x_{N}=1\right]^{T}$ and $\mathbf{e}_{\mathbf{r} i}=[1,0, \ldots, 0]^{T}$ is of dimension $N$.

The second-derivative matrices $P_{l}^{-1} R_{L}$ and $P_{r}^{-1} R_{r}$, as well as the first-derivative matrices $P_{l}^{-1} Q_{l}$ and $P_{r}^{-1} Q_{r}$, are defined as in section 2. The matrices $D_{l}$ and $D_{r}$ are any operators that approximate the first derivative to $O(\Delta x)^{m}$. The obvious first choice would be to use $P_{l}^{-1} Q_{l}$ and $P_{r}^{-1} Q_{r}$, but this choice is not essential for accuracy or stability. (In equation (3.2) we have ignored the physical boundary conditions at $x=1$ and $x=-1$ for the sake of simplicity. )

THEOREM 3.1. Consider the scheme (3.2) for the advection-diffusion equation (3.1). If the matrices $P_{l}, Q_{l}, P_{r}, Q_{r}, R_{L}$ and $R_{r}$ satisfy the first and second derivative properties of section 2 and

$$
\sigma_{3}=\sigma_{1}-a, \quad \sigma_{4}=\sigma_{2}+1, \quad \sigma_{1} \leq \frac{a}{2}-\epsilon\left[\frac{\sigma_{2}^{2}}{4 \alpha_{r}}+\frac{\sigma_{4}^{2}}{4 \alpha_{L}}\right]
$$

then (3.2) is stable.

In the proof which follows, we have without loss of generality considered only the interface terms, and ignored the terms that arise at the physical boundaries. We assume that the physical boundary conditions are implemented by stable and accurate numerical procedures. (See Hesthaven and Gottlicb [2] for a possible implementation).

PROOF: The proof is based on a simple energy estimate. By premultiplying the equations by the vectors $\mathbf{u}^{T}$ and $\mathbf{v}^{T}$, respectively, and adding we obtain

$$
\frac{d}{d t}\left[\|u\|_{P_{l}}^{2}+\|v\|_{P_{r}}^{2}\right]=2 \mathbf{u}^{T}\left(\epsilon R_{l}-a Q_{l}\right) \mathbf{u}+2 \mathbf{v}^{T}\left(\epsilon R_{r}-a Q_{r}\right) \mathbf{v}
$$




$$
\begin{aligned}
& +2 \sigma_{1} u_{i}\left(u_{i}-v_{i}\right)+2 \epsilon \sigma_{2} u_{i}\left[\left(D_{l} u\right)_{i}-\left(D_{r} v\right)_{i}\right] \\
& +2 \sigma_{3} v_{i}\left(v_{i}-u_{i}\right)+2 \epsilon \sigma_{4} v_{i}\left[\left(D_{r} v\right)_{i}-\left(D_{l} u\right)_{i}\right]
\end{aligned}
$$

where $\|u\|_{P_{l}}^{2}=\mathbf{u}^{T} P_{l} \mathbf{u}$, and we have defined $u_{i}, v_{i},\left(D_{l} u\right)_{i}$, and $\left(D_{l} v\right)_{i}$ as $\left.u\right|_{x=x_{i}},\left.v\right|_{x=x_{i}},\left.\left(D_{l} \mathbf{u}\right)\right|_{x=x_{i}}$, and $\left.\left(D_{r} \mathbf{v}\right)\right|_{x=x_{i}}$, respectively. The second-derivative propertics of section 2 lead to

$$
\begin{aligned}
\mathbf{u}^{T} R_{l} \mathbf{u} & \leq-\alpha_{l}\left(D_{l} u\right)_{i}^{2}+u_{i}\left(D_{l} u\right)_{i} \\
\mathbf{v}^{T} R_{\tau} \mathbf{v} & \leq-\alpha_{r}\left(D_{r} v\right)_{i}^{2}-v_{i}\left(D_{\tau} v\right)_{i}
\end{aligned}
$$

where the constants $\alpha_{l}$ and $\alpha_{r}$ are positive.

By using the first-derivative properties of section 2 and equations (3.4) and (3.5) and neglecting the physical boundary terms leads to

$$
\frac{d}{d t}\left[\|u\|_{P_{i}}^{2}+\|v\|_{P_{r}}^{2}\right] \leq \mathbf{w}_{i}^{T} B \mathbf{w}_{i}
$$

where $\mathbf{w}_{i}=\left[u_{i}, v_{i},\left(D_{l} u\right)_{i},\left(D_{r} v\right)_{i}\right]$, and the boundary matrix $B$ defined by

$$
B=\left[\begin{array}{cccc}
\left(-a+2 \sigma_{1}\right) & -\left(\sigma_{1}+\sigma_{3}\right) & \epsilon\left(1+\sigma_{2}\right) & -\epsilon \sigma_{2} \\
-\left(\sigma_{1}+\sigma_{3}\right) & a+2 \sigma_{3} & -\epsilon \sigma_{4} & \epsilon\left(-1+\sigma_{4}\right) \\
\epsilon\left(1+\sigma_{2}\right) & -\epsilon \sigma_{4} & -2 \epsilon \alpha_{l} & 0 \\
-\epsilon \sigma_{2} & \epsilon\left(-1+\sigma_{4}\right) & 0 & -2 \epsilon \alpha_{r}
\end{array}\right]
$$

Straightforward (though tedious) algebra shows that conditions (3.3) yield a non-positive definite matrix $B$, thus proving stability. Details are presented in Appendix I.

In practice, the values of $\sigma_{1}$ through $\sigma_{4}$ are determined as follows. The parameters $\alpha_{r}$ and $\alpha_{l}$ are functions from the numerical method and the chosen grid. The viscous contribution in the constraint equation $\sigma_{1} \leq \frac{a}{2}-\epsilon\left[\frac{\sigma_{2}^{2}}{4 \alpha_{r}}+\frac{\sigma_{1}^{2}}{4 \alpha_{L}}\right]$ is minimized for $\sigma_{2}=\frac{-\alpha_{r}}{\alpha_{r}+\alpha_{l}}$, yielding the expression $\sigma_{1} \leq \frac{a}{2}-\epsilon\left[\frac{1}{4\left(\alpha_{r}+\alpha_{l}\right)}\right]$. The value $\sigma_{1}$ determines the dissipation at the interface, and also influences the effective CFL of the numerical scheme. Values of $\sigma_{1}$ in the range $-1 \leq \sigma_{1} \leq 0$ provide a compromise between adequate levels of dissipation, and acceptable numerical efficiency.

We have shown that the linking of two domains at an interface with the interface conditions prescribed in Theorem 3.1 is stable in a semidiscrete sense for specific values of the penalty parameters $\sigma_{1}$ through $\sigma_{4}$. The basic methodology can be extended to an arbitrary number of subdomains without complication. The only constraint is that the numerical method must satisfy the SBP framework. The methodology does not rely on subdomain size and does not require the same SBP operator to be used in each domain. In principle, a finite-difference operator of any order, as well as spectral operators on subdomains of arbitrary size, can be linked together in a stable manner. Practical details on how to chose $\sigma_{1}$ through $\sigma_{4}$ are included in the results section (Section 6).

In section 2, we presented the general form of second-derivative operators appropriate for this work. We then noted two specific derivative operators that satisfy this form. We now show that both choices for the matrices $R_{l}$ (and $R_{r}$ ) suggested in section 2 satisfy conditions (3.4) and (3.5) of Theorem 3.1. We start with the first option (i.e. $R_{l}=Q_{l} P_{l}^{-1} Q_{l}$ ). In this case, the first derivative matrix in (3.2) is $D_{l}=P_{l}^{-1} Q_{l}$. Thus, the quantity $\mathbf{u}^{T} R_{l} \mathbf{u}$ becomes

$$
\begin{aligned}
\mathbf{u}^{T} Q_{l} P_{l}^{-1} Q_{l} \mathbf{u} & =\mathbf{u}^{T} Q_{l} P_{l}^{-1} P_{l} P_{l}^{-1} Q_{l} \mathbf{u} \\
& =-\left(P_{l}^{-1} Q_{l} \mathbf{u}\right)^{T} P_{l}\left(P_{l}^{-1} Q_{l} \mathbf{u}\right)+u_{i}\left(P_{l}^{-1} Q_{l} u\right)_{i}
\end{aligned}
$$


where we have used the SBP property $Q+Q^{T}=D$, and have ignored the physical boundary contribution.

We recall now that $P_{l} \geq(\Delta x) p_{l}$ so that

$$
\begin{aligned}
\mathbf{u}^{T} R_{l} \mathbf{u} & =\mathbf{u}^{T} Q_{l} P_{l}^{-1} Q_{l} \mathbf{u} \\
& \leq-(\Delta x) p_{l}\left|\left(D_{l} \mathbf{u}\right)\right|^{2}+u_{i}\left(D_{l} u\right)_{i}
\end{aligned}
$$

Thus, (3.4) is satisfied with $\alpha_{l}=(\Delta x) p_{l}$. A similar result holds for $R_{r}$ with $\alpha_{r}=(\Delta x) p_{r}$.

The second choice presented in section 2 for the sccond-derivative operator $P^{-1} R_{l}$ is of the form of equation (2.3):

$$
P^{-1} R_{l}=P^{-1}\left(-S^{T} M+D\right) S
$$

For the purpose of proving stability, we relate the two matrices $D_{l}=S$. (In actuality, only the first and last rows satisfy $D_{l}=S$. They are, however, the only portions of the matrices that enter the proof.)

$$
\begin{aligned}
\mathbf{u}^{T} R_{l} \mathbf{u} & =-(S \mathbf{u})^{T} M S \mathbf{u}+U_{i}(S \mathbf{u})_{i} \\
& \leq-(\Delta x) m|S \mathbf{u}|^{2}+U_{i}(S \mathbf{u})_{i}
\end{aligned}
$$

Thus, (3.4) is satisfied with $\alpha_{l}=(\Delta x) m$.

4. Conservation at the Interface. The Lax-Wendroff theorem [12] addresses the complexities encountered in solving nonlinear conservation laws. The theorem states that a convergent numerical approximation $U_{l}(x, t)$, computed with a consistent and conservative method, converges to a weak solution of the conservation law. Note that discrete conservation is necessary to satisfy the conditions of the theorem.

A heuristic definition of conservation (commonly encountered by practitioners) describes how the numerical flux function "telescopes" across a domain to the boundaries. The total quantity of a conserved variable in any region changes only as a result of the flux through the boundaries of the region. We, however, rely on a broader definition of conservation motivated by the original proof of the Lax-Wendroff theorem. We demand that the numerical flux telescope across the domain, and that all moments of the flux against an arbitrary test function telescope across the domain. This additional constraint demands an equivalence between the weak forms of the continuous and discrete operators.

We begin by discussing conservation in a single domain. Consider the nonlinear equation $U_{t}+F_{x}=0$ on $-1 \leq x \leq 1$ and $t \geq 0$. Note that in the linear case $F=a U$ and we obtain (3.1) with $\epsilon=0$. To obtain the weak form of this equation we multiply by an arbitrary test function $\phi(x, t)$ that vanishes on the boundaries. By integrating with respect to space and time we obtain an integral statement of the original differential equation:

$$
\left.\int_{-1}^{1} \phi U d x\right|_{0} ^{t}-\int_{0}^{t} \int_{-1}^{1}\left(U \phi_{t}+F \phi_{x}\right) d x d \tau=0
$$

Now consider the semidiscrete equation given by $P \mathbf{U}_{t}+Q \mathbf{F}=0$. Here, we have replaced the spatial derivative $F_{x}$ in the continuous case with an SBP derivative operator of order $(\Delta x)^{r}$. By multiplying by the discrete vector $\phi\left(x_{j}\right)=\phi^{T}$ (the discrete analog of integration) and integrating with respect to time, we obtain

$$
\left.\phi^{T} P U\right|_{0} ^{t}-\int_{0}^{t}\left(U^{T} P \phi_{t}+F^{T} Q \phi\right) d \tau=0
$$

Thus, the semi-discrete operator satisfies a weak form similar to that of the continuous operator, and asymptotically approaches the continuous operator in the limit of infinite spatial resolution. The special form of the 
$P$ and $Q$ matrices present in the SBP operators enables the semidiscrete operator to mimic the conservation property of the continuous operator.

The equivalence between the continuous and semi-discrete operators is more more complicated for multiple domains. The conservation property of the SBP operator does not necessarily apply at an interface boundary. Under very mild restrictions, however, the SBP interface operators telescope out to the physical boundaries, as does the continuous operator. Because conservation is only necessary for the advection terms in the advection-diffusion equation, we set $\epsilon=0$ (see equation (3.1)) and prove conservation for a two-domain discretization. We prove conservation for a general nonlinear flux. Note that the penalty parameters for this nonlinear case are designated $\hat{\sigma}_{1}$ and $\hat{\sigma}_{3}$. The resulting conservation condition obtained in the nonlinear case is slightly different from that obtained in the linear analysis. This difference results from different scalings of the penalty parameters.

THEOREM 4.1. Assume the nonlinear equation $\frac{\partial U}{\partial t}+\frac{\partial F(U)}{\partial x}=0$ is valid on the interval $-1 \leq x \leq$ $1, t>0$, divided arbitrarily into two subintervals $-1 \leq x \leq x_{i}$ and $x_{i} \leq x \leq 1$. On each subinterval, a discretization is used that satisfies the SBP framework, and boundary conditions are imposed via penalties in the form

$$
\begin{aligned}
& \mathbf{u}_{t}+P_{l}^{-1} Q_{l} \mathbf{F}(\mathbf{u})=\hat{\sigma}_{1} P_{l}^{-1} \mathbf{e}_{1_{i}}\left[F\left(u\left(x_{i}\right)\right)-F\left(v\left(x_{i}\right)\right)\right] \\
& \mathbf{v}_{t}+P_{r}^{-1} Q_{r} \mathbf{F}(\mathbf{v})=\hat{\sigma}_{3} P_{r}^{-1} \mathbf{e}_{\mathbf{r} i}\left[F\left(v\left(x_{i}\right)\right)-F\left(u\left(x_{i}\right)\right)\right]
\end{aligned}
$$

where $\mathbf{u}=\left[u_{0}(t), u_{1}(t), \ldots, u_{M}(t)\right]^{T}$ is defined in the left domain at the points $\mathbf{x}_{\mathbf{L}}=\left[x_{0}=-1, x_{1}, \ldots, x_{M}=x_{i}\right]^{T}$. and $\mathbf{e}_{1_{i}}=[0, \ldots, 0,1]^{T}$ is of dimension $M$, with similar definitions on the right domain. The discretization is conservative provided that the stability condition $\hat{\sigma}_{3}=\hat{\sigma}_{1}-1$ is satisfied.

PROOF: For multiple domains, we proceed as shown previously in the single-domain case. Multiplying equations (4.1) by the vectors $\phi^{T} P_{l}$ and $\phi^{T} P_{r}$, respectively, yields the set of equations

$$
\begin{aligned}
& \phi^{T} P_{l} \mathbf{u}_{t}+\phi^{T} Q_{l} \mathbf{F}(\mathbf{u})=\hat{\sigma}_{1} \phi\left(x_{i}\right)\left(F\left(u\left(x_{i}\right)\right)-F\left(v\left(x_{i}\right)\right)\right) \\
& \phi^{T} P_{r} \mathbf{v}_{t}+\phi^{T} Q_{r} \mathbf{F}(\mathbf{v})=\hat{\sigma}_{3} \phi\left(x_{i}\right)\left(F\left(v\left(x_{i}\right)\right)-F\left(u\left(x_{i}\right)\right)\right)
\end{aligned}
$$

Using the properties of $Q_{l}$ and $Q_{r}$ we get

$$
\begin{gathered}
\phi^{T} P_{l} \mathbf{u}_{t}-\mathbf{F}^{T} Q_{l} \phi+\phi\left(x_{i}\right) F\left(u\left(x_{i}\right)\right)=\hat{\sigma}_{1} \phi\left(x_{i}\right)\left(F\left(u\left(x_{i}\right)\right)-F\left(v\left(x_{i}\right)\right)\right) \\
\phi^{T} P_{r} \mathbf{v}_{t}-\mathbf{F}^{T} Q_{r} \phi-\phi\left(x_{i}\right) F\left(v\left(x_{i}\right)\right)=\hat{\sigma}_{3} \phi\left(x_{i}\right)\left(F\left(v\left(x_{i}\right)\right)-F\left(u\left(x_{i}\right)\right)\right)
\end{gathered}
$$

By integrating with respect to time and making use of the fact that $\phi$ is continuous at the interface, we get

$$
\begin{aligned}
\left.\phi^{T} P_{l} \mathbf{u}\right|_{0} ^{t}+\left.\phi^{T} P_{r} \mathbf{v}\right|_{0} ^{t} & =\int_{0}^{t}\left(\mathbf{u}^{T} P_{l} \phi_{t}+\mathbf{F}^{T} Q_{l} \phi\right) d \tau \\
& +\int_{0}^{t}\left(\mathbf{v}^{T} P_{r} \phi_{t}+\mathbf{F}^{T} Q_{r} \phi\right) d \tau \\
& +\int_{0}^{t} \phi_{i} F\left(u\left(x_{i}\right)\right)\left(\hat{\sigma}_{1}-\hat{\sigma}_{3}-1\right) d \tau \\
& +\int_{0}^{t} \phi_{i} F\left(v\left(x_{i}\right)\right)\left(\hat{\sigma}_{3}-\hat{\sigma}_{1}+1\right) d \tau
\end{aligned}
$$

Obviously, the condition $\hat{\sigma}_{3}=\hat{\sigma}_{1}-1$ eliminates the interface terms from the expression and leaves the desired weak form of the semidiscrete equation. Thus, the theorem is proved. 
5. Accuracy of Boundary Conditions. A significant obstacle in dealing with high-order finitedifference schemes is the formulation of stable stencils near the boundaries. A uniformly high-order approximation should be maintained if possible up to the boundary. In most high-order formulations, ensuring uniform accuracy up to the boundaries is difficult when numerical stability must be maintained. Fortunately, Gustafsson [13] showed that difference approximations to mixed hyperbolic parabolic equations admit global design accuracy when a finite number of points (independent of $N$ ) are closed with stencils that are less accurate by 1 order. For example, a fourth-order interior discretization will asymptotically recover fourth-order $L_{2}$ accuracy with third-order closures near the boundaries.

In this section, we confirm that the physical boundary conditions must be imposed with at least the design accuracy in the context of interface boundary conditions. We begin by inspecting equation (3.2) and by defining the truncation error as that error committed by substituting the exact solution into the scheme. Denote by $\mathbf{V}_{1}$ and $\mathbf{V}_{\mathbf{r}}$ the projection of the exact solution in the two domains. Substituting the exact solution into the first equation in (3.2) yields

$$
P_{l} \mathbf{T}_{l_{e}}=P_{l} \frac{\partial \mathbf{V}_{1}}{\partial t}+a Q_{l} \mathbf{V}_{\mathbf{l}}-\epsilon R_{l} \mathbf{V}_{\mathbf{l}}+\sigma_{1} \mathbf{e}_{\mathbf{l}_{i}}\left(\mathbf{V}_{\mathbf{l}_{x=x_{\mathbf{i}}}}-\mathbf{V}_{\mathbf{r} x=x_{i}}\right)+\sigma_{2} \mathbf{e}_{1_{i}}\left(\left.\left(D_{l} \mathbf{V}_{1}\right)\right|_{x=x_{i}}-\left.\left(D_{r} \mathbf{V}_{\mathbf{r}}\right)\right|_{x=x_{i}}\right)
$$

with a similar expression in the right domain. The differentiation matrices are accurate to the design order of the method. Thus, the first three terms to the right of the equality, reduce to the truncation crror of the spatial approximation. (Except for a finite number of points that are lower by 1 order near the interfaces and the physical boundary). Examining the truncation error from the penalty terms, we observe that $V$ is smooth across the interface, and $\mathbf{V}_{\mathbf{l}_{i}}-\mathbf{V}_{\mathbf{r} i}=0$. Thus, we only need that $D_{l} \mathbf{V}_{\mathbf{l}_{x=x_{i}}}$ and $D_{r} \mathbf{V}_{\mathbf{r} x=x_{i}}$ approximate the first derivative to the design order of accuracy. The exact nature of the solution error near the boundaries is extremely complicated due to the points treated less accurately in that vicinity. More details will be presented in a future work on this subject. We show by numerical example, however, that order reduction occurs when the interface derivative is treated with less than design accuracy. (See Table 5).

5.1. Uniform Grid.. Now we demonstrate that the physical boundary conditions must be imposed with accuracy of at least design order to maintain global design accuracy. This condition is a natural consequence of the overall dependence of the solution on the boundary conditions. The test problem we use is the Burgers' equation

$$
\frac{\partial U}{\partial t}+U \frac{\partial U}{\partial x}=\epsilon \frac{\partial^{2} U}{\partial x^{2}} \quad-1 \leq x \leq 1, t>0
$$

with the exact solution

$$
U(x, t)=-a \tanh \left(a \frac{x-c t}{2 \epsilon}\right)+c,-\infty<x<\infty, t<0 .
$$

The solution of (5.1) requires imposition of boundary conditions at each end of the physical domain. We choose Robin boundary conditions of the form

$$
\alpha u(-1, t)-\left.\beta \frac{\partial u}{\partial x}\right|_{-1}=g_{-1}(t) ; \quad \gamma u(1, t)-\left.\delta \frac{\partial u}{\partial x}\right|_{1}=g_{1}(t)
$$

such that the problem is mathematically well-posed. (See Hesthaven and Gottlieb [2] for the constraints on $\alpha, \beta, \gamma$, and $\delta$ ). The physical boundary conditions were imposed in penalty form, as described in the work of Hesthaven and Gottlieb [2]. The time-advancement scheme is the five-stage fourth-order low-storage Runge-Kutta scheme. The time step was chosen to ensure that the temporal error in the formulation was 
small relative to the spatial error. The simulation is run to a physical time of $T=1$, and the viscosity is determined by the value $\epsilon=510^{-1}$.

Tables 1 to 4 show the results of a grid-refinement study on a single domain with a fourth-order explicit interior scheme. The accuracy of the boundary closure and of the physical boundary condition are parameters in the study. Table 2 shows the results of the refinement study with a uniformly fourth-order-accurate scheme $(4,4-4-4,4)$ with the derivative term in the Robins' boundary conditions approximated to $O\left(\Delta x^{4}\right)$. We note

TABLE 5.1

$L_{2}$ Solution Errors: Convergence rate of uniformly fourth-order sch eme

\begin{tabular}{ccc}
\hline$N$ & $L O G_{10}$ error & Rate \\
\hline 33 & -3.847 & \\
65 & -4.082 & 2.31 \\
129 & -5.239 & 3.84 \\
257 & -6.486 & 4.14 \\
513 & -7.731 & 4.14 \\
1025 & -8.960 & 4.87 \\
\hline
\end{tabular}

that the convergence rate in Table 1 is fourth order and that the design accuracy is achieved.

Table 2 shows the sccond study in which boundary closure accuracy is relaxed by one order. The resulting scheme (3,3-4-3,3) is third order locally at each boundary and fourth order in the interior. (Both the inviscid and viscous stencils are reduced by one order of accuracy near the boundaries.) The physical boundary condition is still approximated to $O\left(\Delta x^{4}\right)$.

TABLE 5.2

$L_{2}$ Solution Errors: Convergence rate of fourth-order scheme with third-order closure at boundaries.

\begin{tabular}{ccc}
\hline$N$ & LOG $_{10}$ error & Rate \\
\hline 33 & -3.694 & \\
65 & -4.797 & 3.66 \\
129 & -5.971 & 3.90 \\
257 & -6.117 & 3.81 \\
513 & -7.276 & 3.85 \\
1025 & -9.455 & 3.92 \\
\hline
\end{tabular}

We note that the convergence rate in Table 2 asymptotes to fourth order and that the absolute levels of error are comparable to those obtained using the (4,4-4-4,4) scheme. Again, design accuracy is achicved.

Table 3 shows the third study, in which boundary closure accuracy is relaxed by two orders. The resulting scheme $(2,2-4-2,2)$ is second order locally at each boundary and fourth order in the interior. (Only the viscous terms are reduced by two orders of accuracy near the boundaries.) The physical boundary condition is still approximated to $O\left(\Delta x^{4}\right)$.

We note that the convergence rate in Table 3 asymptotes to third order, which is a reduction in global accuracy of one order. This behavior is consistent with Gustafsson's [13] theory, specifically, that global solution accuracy allows a finite number of stencils to be reduced by one order of accuracy.

Table 4 shows the final study, in which boundary closure accuracy is uniformly fourth-order accurate $(4,4-4-4,4)$. The physical boundary condition is approximated to $O\left(\Delta x^{3}\right)$, however. The convergence rate in 
TABLE 5.3

$L_{2}$ Solution Errors: Convergence rate of fourth-onder scheme with second-onder closure at boundaries.

\begin{tabular}{ccc}
\hline$N$ & LOG $_{10}$ error & Rate \\
\hline 33 & -2.974 & \\
65 & -4.074 & 3.65 \\
129 & -5.519 & 4.80 \\
257 & -6.284 & 2.54 \\
513 & -7.048 & 2.54 \\
1025 & -7.898 & 2.82 \\
\hline
\end{tabular}

Table 4 asymptotes to third order, which is a reduction in global accuracy by one order.

TABLE 5.4

$L_{2}$ Solution Errors: Convergence rate of uniformly fourth-order scheme, using third-order accurate boundary conditions.

\begin{tabular}{ccc}
\hline$N$ & LOG $_{10}$ error & Rate \\
\hline 33 & -3.004 & \\
65 & -4.002 & 3.32 \\
129 & -4.764 & 2.53 \\
257 & -5.636 & 2.90 \\
513 & -6.531 & 2.97 \\
1025 & -7.898 & 2.82 \\
\hline
\end{tabular}

This series of tests on the single domain indicates the need to impose the physical boundary condition with design accuracy. However, closing the near boundary stencils with an accuracy that is one order less than the design interior accuracy appears to be sufficient. A similar conclusion was reached with a second-order-accurate scheme (1-2-1) and second-order physical boundary conditions.

We now demonstrate by numerical example that these results generalize to the case of multiple domains. Table 5 shows a grid-refinement study that compares one and eight spatial domains. The numerical test problem is the previously described Burgers' equation using a value of $\epsilon=10^{-2}$. The numerical scheme used in both cases is the $(3,3,3,3-4-3,3,3,3)$ scheme with physical boundary conditions imposed to an accuracy of $O\left(\Delta x^{4}\right)$.

TABLE 5.5

$L_{2}$ Solution Errors: Convergence rate of fourth-order scheme with third-order closure at interfaces, on multiple domain problem.

\begin{tabular}{rcccc}
\hline & 1 domains & \multicolumn{3}{c}{8 domains } \\
\hline$N$ & LOG ${ }_{10}$ error & Rate & LOG ${ }_{10}$ error & Rate \\
\hline 97 & -2.148 & & -2.125 \\
193 & -3.016 & 2.88 & -3.143 & 3.38 \\
385 & -4.214 & 3.98 & -4.485 & 4.45 \\
769 & -5.372 & 3.85 & -5.656 & 3.38 \\
1537 & -6.505 & 3.76 & -6.866 & 4.02 \\
3063 & -7.664 & 3.85 & -8.055 & 3.95 \\
\hline
\end{tabular}


We note that the convergence rate in Table 5 asymptotes to fourth order, for both the one- and eightdomain cases. This example demonstrates that design accuracy is achicved with multiple domains so long as the physical boundary conditions are imposed with design accuracy and the numerical closures near the interfaces are at most one order of accuracy less than the design accuracy of the interior scheme.

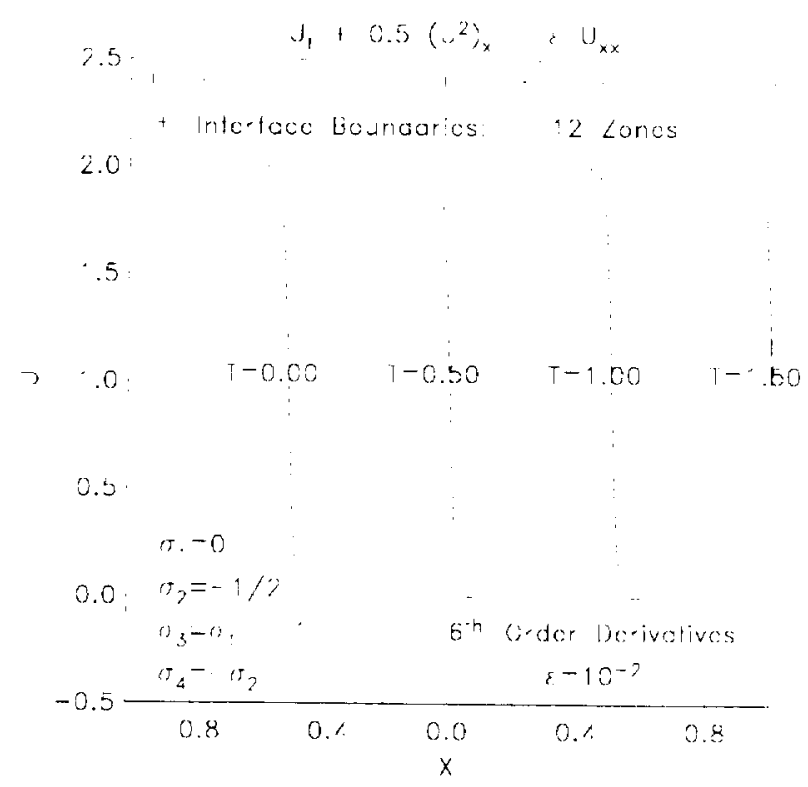

FIG. 5.1. The Burgers equation solved using a sixth-order scheme with randomly generated interface points.

5.2. Nonuniform Domain. The final problem we solve is the nonlinear Burgers' equation with unequally spaced subdomains and a sixth-order scheme. Details of the numerical discretization are included in the appendix. The Burgers' equation in the form of equation (5.1) is solved throughout the domain with a viscosity parameter of $\epsilon=10^{-2}$. The domain is divided into 12 subdomains, each with the same number of points and a uniform local discretization. The domain interfaces are placed randomly throughout the domain. The ratio of maximum to minimum subdomain size is about 15:1. Figure 1 shows the solution at four different times. The "symbols" at the top of the figure show the positions of the 11 interface points. The profiles are smooth and monotone for this discretization. Figure 2 shows the logarithm of the solution error plotted as a function of space on the sequence of five grids.

This problem demonstrates the stability and accuracy of the new interface treatments. The discretizations asymptote to a convergence rate of sixth order on the sequence of grids. Table 6 shows the convergence rate of the calculations, for two different values of the parameter $\epsilon$. The steep gradients are resolved to high-order on all grids for $\epsilon=10^{-2}$. For $\epsilon=210^{-3}$, the two coarsest grids are not yet achieving high-order accuracy, and two-point grid oscillations exist in the solution. Further reduction of $\epsilon$ causes numerical instability, emanating from the interface location, as the gradients pass the interface. Increasing the robustness of the interface conditions for marginally resolved/discontinuous cases is the focus of current research.

6. Conclusions. We focus on high-order finite difference schemes, which satisfy the summation-byparts (SBP) discretization framework. We show stable and conservative interface treatments of arbitrary spatial accuracy for the linear advection-diffusion equation. Problems with multiple domains and abruptly changing mesh sizes are considered. 


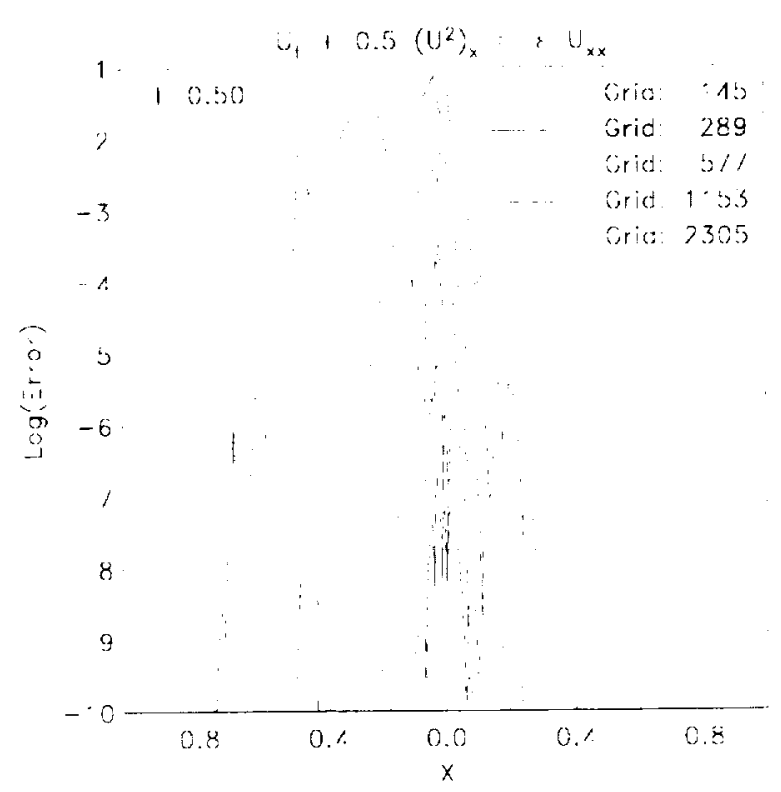

FIG. 5.2. Errors obtained from Burgers equation solved on a sequence of grids with a sixth-order scheme.

TABLE 5.6

$L_{2}$ Solution Errors: Convergence of sixth-onder scheme unth twelve subdomains and interfaces distributed randomly.

\begin{tabular}{rcccc}
\hline & $\epsilon=10^{-2}$ & \multicolumn{3}{c}{$\epsilon=210^{-3}$} \\
$N$ & LOG ${ }_{10}$ error & Rate & LOG ${ }_{10}$ error & Rate \\
\hline 145 & -3.090 & & -1.376 & \\
289 & -4.641 & 5.15 & -1.865 & 1.62 \\
577 & -5.915 & 4.22 & -3.053 & 3.95 \\
1153 & -7.520 & 5.33 & -4.574 & 5.05 \\
2305 & -9.370 & 6.15 & -5.834 & 4.18 \\
\hline
\end{tabular}

Finite-difference operators are shown to admit design accuracy ( $p^{\text {th }}$-order global accuracy) when $p-1^{\text {th }}$ order stencil closures are used near boundaries if the physical boundary conditions are imposed with $p^{\text {th }}$-order accuracy. Finite-difference operators of up to sixth order are constructed which satisfy the constraints of the new interface procedures.

Accurate sixth order calculations are achieved for the nonlinear Burgers equation on a twelve subdomain problem having randomly distributed interfaces.

\section{REFERENCES}

[1] D. A. Kopriva, Spectral methods for the Euler equations-The blunt body problem revisited, AIAA Journal, 29, (1991), pp. 1458-1462.

[2] J. S. Hesthaven And D. Gottlieb, A Stable Penalty Method for the Compressible Navier-Stokes Equations: I. Open Boundary Conditions, SIAM J. Sci. Comput. 17, 3, (1996), pp 579-612.

[3] H.-O. KREISS AND G. SCHERER, Finite element and finite difference methods for hyperbolic partial differential equations, Mathematical Aspects of Finite Elements in Partial Differential Equations, 
Acadcmic Press, New York, (1974).

[4] P. Olsson, High-order difference methods and data-parallel implementation, $\mathrm{PhD}$ Thesis, Uppsala University, Department of Scientific Computing, (1992).

[5] P. Olsson, Summation by Parts, Projections, and Stability I, Math. Comp., 64, (1995) pp. 1035-1065.

[6] P. Olsson, Summation by Parts, Projections, and Stability II, Math. Comp., 64, (1995) pp. 1473-1493.

[7] B. Strand, High-Order Difference Approximations for Hyperbolic Initial Boundary Value Problems, PhD Thesis, Uppsala University, Department of Scientific Computing, (1996).

[8] B. Strand, Summation by Parts for Finite Difference Approximations for $d / d x$, J. Comp. Phy., 110, No. 1, (1994), pp. 47-67.

[9] M. H. Carpenter, D. Gottlieb, and S. Abarbanel, The Stability of Numerical Boundary Treatments for Compact High-Order Finite-Difference Schemes, J. Comp. Phy., 108, No. 2, (1993), pp. 272-295.

[10] M. H. Carpenter and D. Gottlieb, Spectral Methods on Arbitrary Grids, J. Comp. Phy., 129, No. 0234, (1996), pp. 74-86.

[11] M. H. Carpenter and J. Otto, High-Order Cyclo-Difference Techniques: An Alternative to Finite Differences J. Comp. Phy., 118, (1995), pp. 242-260.

[12] P. D. Lax and B. Wendroff, Systems of Conservation Laws, Comm. Pure Appl. Math, 13, (1960), pp. 217-237.

[13] B. Gustafsson, The Convergence Rate for Difference Approximations to Mixed Initial Boundary Value Problems, Math. Comp. 29, 130, (1975), pp. 396-406.

Appendix I. Stability. Here we show the algebra involved in proving THEOREM 3.1. We begin by restating of the stability condition presented in equation (3.6), governing the total energy of the system:

$$
\frac{d}{d t}\left[\|u\|_{P_{l}}^{2}+\|v\|_{P_{r}}^{2}\right] \leq \mathbf{w}_{i}^{T} B \mathbf{w}_{i}
$$

where $\mathbf{w}_{i}=\left[u_{i}, v_{i},\left(D_{l} u\right)_{i},\left(D_{r} v\right)_{i}\right]$, and the boundary matrix defined in equation (3.7) is defined by

$$
B=\left[\begin{array}{cccc}
\left(-a+2 \sigma_{1}\right) & -\left(\sigma_{1}+\sigma_{3}\right) & \epsilon\left(1+\sigma_{2}\right) & -\epsilon \sigma_{2} \\
-\left(\sigma_{1}+\sigma_{3}\right) & a+2 \sigma_{3} & -\epsilon \sigma_{4} & \epsilon\left(-1+\sigma_{4}\right) \\
\epsilon\left(1+\sigma_{2}\right) & -\epsilon \sigma_{4} & -2 \epsilon \alpha_{l} & 0 \\
-\epsilon \sigma_{2} & \epsilon\left(-1+\sigma_{4}\right) & 0 & -2 \epsilon \alpha_{r}
\end{array}\right]
$$

The stability of this matrix is easicr to analyze if it is rotated with a similarity transformation. Define the new vector $\hat{\mathbf{w}}=S \mathbf{w}$ such that:

$$
\hat{\mathbf{w}}=\frac{1}{\sqrt{2}}\left[\begin{array}{c}
u_{i}-v_{i} \\
u_{i}+v_{i} \\
\left(D_{l} u\right)_{i}-\left(D_{r} v\right)_{i} \\
\left(D_{l} u\right)_{i}+\left(D_{r} v\right)_{i}
\end{array}\right]=\frac{1}{\sqrt{2}}\left[\begin{array}{cccc}
1 & -1 & 0 & 0 \\
1 & 1 & 0 & 0 \\
0 & 0 & 1 & -1 \\
0 & 0 & 1 & 1
\end{array}\right]\left[\begin{array}{c}
u_{i} \\
v_{i} \\
\left(D_{l} u\right)_{i} \\
\left(D_{r} v\right)_{i}
\end{array}\right]
$$

The similarity rotation matrix has the property $S^{T}=S^{-1}$ as can easily be verified. The rotation matrix $S$ can be used to transform the stability condition defined by equation (3.6) into the following equivalent condition:

$$
\mathbf{w}_{i}^{T} M^{i} \mathbf{w}_{i}=\mathbf{w}_{i}^{T} S^{T} S M^{i} S^{T} S \mathbf{w}_{i}=\hat{\mathbf{w}}^{T} \hat{M}^{i} \hat{\mathbf{w}} \leq 0
$$


where

$$
\hat{M}^{i}=\left[\begin{array}{cccc}
2\left(\sigma_{1}+\sigma_{3}\right) & -\left(-\sigma_{1}+\sigma_{3}+a\right) & \epsilon\left(\sigma_{2}+\sigma_{4}\right) & \epsilon \\
-\left(-\sigma_{1}+\sigma_{3}+a\right) & 0 & -\epsilon\left(-\sigma_{2}+\sigma_{4}-1\right) & 0 \\
\epsilon\left(\sigma_{2}+\sigma_{4}\right) & -\epsilon\left(-\sigma_{2}+\sigma_{4}-1\right) & -\epsilon\left(\alpha_{r}+\alpha_{l}\right) & \epsilon\left(\alpha_{r}-\alpha_{l}\right) \\
\epsilon & 0 & \epsilon\left(\alpha_{r}-\alpha_{l}\right) & -\epsilon\left(\alpha_{r}+\alpha_{l}\right)
\end{array}\right]
$$

To ensure negative definiteness, every sub-matrix in the matrix $\hat{M}^{i}$ must be negative definite. We observe by inspection that $\left(\sigma_{1}+\sigma_{3}\right) \leq 0$ is a necessary condition. Analyzing the $2 x 2$ sub-matrices along the diagonal, we obtain the necessary conditions $\left(-\sigma_{1}+\sigma_{3}+a\right)=0$, and $\epsilon\left(-\sigma_{2}+\sigma_{4}-1\right)=0$. Substituting the equalities $\left(-\sigma_{1}+\sigma_{3}+a\right)=0$ and $\left(-\sigma_{2}+\sigma_{4}-1\right)=0$ into the matrix $\hat{M}^{i}$ yields:

$$
\hat{M}^{i}=\left[\begin{array}{cccc}
2\left(2 \sigma_{1}-a\right) & 0 & \epsilon\left(2 \sigma_{2}+1\right) & \epsilon \\
0 & 0 & 0 & 0 \\
\epsilon\left(2 \sigma_{2}+1\right) & 0 & -\epsilon\left(\alpha_{r}+\alpha_{l}\right) & \epsilon\left(\alpha_{r}-\alpha_{l}\right) \\
\epsilon & 0 & \epsilon\left(\alpha_{r}-\alpha_{l}\right) & -\epsilon\left(\alpha_{r}+\alpha_{l}\right)
\end{array}\right]
$$

A symmetric matrix can be rotated into diagonal form by an orthogonal matrix, making the condition of negative semi-definiteness

$$
\hat{\mathbf{w}}^{T} \hat{U}^{T} D^{i} \hat{U} \hat{\mathbf{w}} \leq \mathbf{0}
$$

where $\hat{U}$ is the orthogonal matrix that satisfies $\hat{U}^{T} D^{i} \hat{U}=\hat{M}^{i}$. Pre- and post- multiplication of $\hat{M}^{i}$ by suitable rotation matrices $M_{\lambda}=R_{1}^{T} \hat{M}^{i} R_{1}$, yield the equivalent condition

$$
\hat{\mathbf{w}}^{T} R_{1}^{T} \hat{U}^{T} D^{i} \hat{U} R_{1} \hat{\mathbf{w}} \leq 0
$$

The matrix $R_{1}$, chosen to yield a diagonal expression for the matrix $M_{\lambda}$ is

$$
L_{1}=\left[\begin{array}{cccc}
1 & 0 & 0 & 0 \\
0 & 1 & 0 & 0 \\
L_{3,1} & 0 & 1 & 0 \\
L_{4,1} & 0 & L_{4,3} & 1
\end{array}\right]
$$

with

$$
\begin{aligned}
& L_{3,1}=\frac{-\epsilon\left(2 \sigma_{2}+1\right)}{2\left(2 \sigma_{1}-a\right)} \\
& L_{4,1}=\frac{-2 \epsilon\left(\alpha_{r} \sigma_{2}-\alpha_{l} \sigma_{2}+\alpha_{r}\right)}{\epsilon\left(4 \sigma_{2}^{2}+4 \sigma_{2}+1\right)+\left(4 \sigma_{1}-2 a\right)\left(\alpha_{r}+\alpha_{l}\right)} \\
& L_{4,3}=\frac{-\left(\epsilon\left(2 \sigma_{2}+1\right)+\left(-4 \sigma_{1}+2 a\right)\left(\alpha_{r}-\alpha_{l}\right)\right)}{\epsilon\left(4 \sigma_{2}^{2}+4 \sigma_{2}+1\right)+\left(4 \sigma_{1}-2 a\right)\left(\alpha_{r}+\alpha_{l}\right)}
\end{aligned}
$$

The diagonal elements of $M_{\lambda}$ are

$$
\lambda_{1}=2\left(2 \sigma_{1}-a\right)
$$




$$
\begin{aligned}
& \lambda_{2}=0 \\
& \lambda_{3}=\frac{-\epsilon\left(\epsilon\left(4 \sigma_{2}^{2}+4 \sigma_{2}+1\right)+\left(4 \sigma_{1}-2 a\right)\left(\alpha_{r}+\alpha_{l}\right)\right)}{2\left(2 \sigma_{1}-a\right)} \\
& \lambda_{4}=\frac{-4 \epsilon\left(\alpha_{r} \epsilon\left(\sigma_{2}+1\right)^{2}+\alpha_{l} \epsilon \sigma_{2}^{2}+\left(4 \sigma_{1}-2 a\right) \alpha_{l} \alpha_{r}\right)}{\epsilon\left(4 \sigma_{2}^{2}+4 \sigma_{2}+1\right)+\left(4 \sigma_{1}-2 a\right)\left(\alpha_{\tau}+\alpha_{l}\right)}
\end{aligned}
$$

These eigenvalues must be less than or equal to zero to ensure stability of the interface condition. The resulting condition of stability is

$$
\sigma_{1} \leq \frac{a}{2}-\epsilon\left[\frac{\sigma_{2}^{2}}{4 \alpha_{r}}+\frac{\sigma_{4}^{2}}{4 \alpha_{L}}\right]
$$

Combining this expression with the constraints $\sigma_{3}=\sigma_{1}-a$ and $\sigma_{4}=\sigma_{2}+1$ yield the conditions of THEOREM 3.1.

Appendix II. Stencils. We now present the specific form of the stencils that satisfy the SBP stability requirements, and the accuracy requirements shown necessary in the previous numerical study. At second order, the discretization matrix for the advection terms that satisfy the constraint $A_{1}=P^{-1} Q$ is

$$
A=\frac{1}{2 \Delta x}\left[\begin{array}{ccccccc}
-2 & 2 & & & & & \\
-1 & 0 & 1 & & & & \\
& \cdot & \cdot & \cdot & & & \\
& & \cdot & \cdot & \cdot & & \\
& & & \cdot & \cdot & \cdot & \\
& & & & -1 & 0 & 1 \\
& & & & & -2 & 2
\end{array}\right]
$$

where

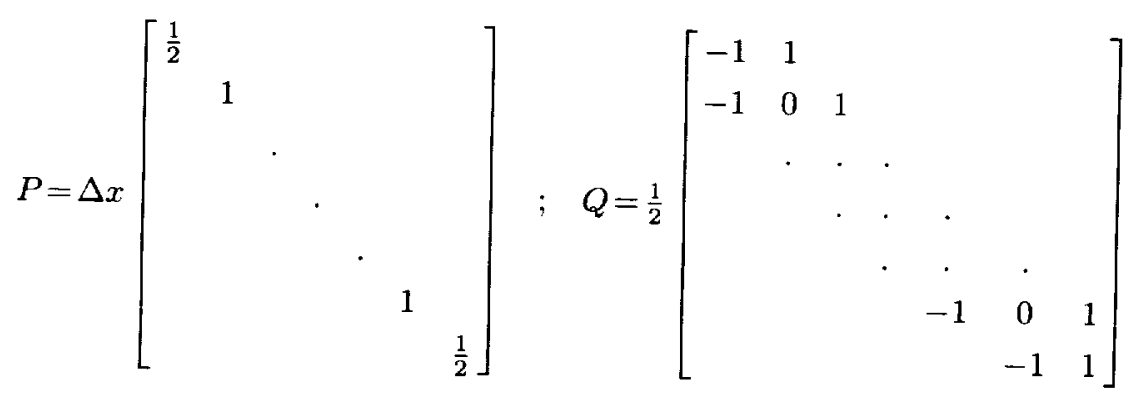
is

The discretization matrix for the diffusion terms that satisfies the constraint $A_{2}=P^{-1}\left(-S^{T} R+D\right) S$

$$
A=\frac{1}{(\Delta x)^{2}}\left[\begin{array}{ccccccc}
1 & -2 & 1 & & & & \\
1 & -2 & 1 & & & & \\
& \cdot & \cdot & \cdot & & & \\
& & \cdot & \cdot & \cdot & & \\
& & & \cdot & \cdot & \cdot & \\
& & & & 1 & -2 & 1 \\
& & & & 1 & -2 & 1
\end{array}\right]
$$


where

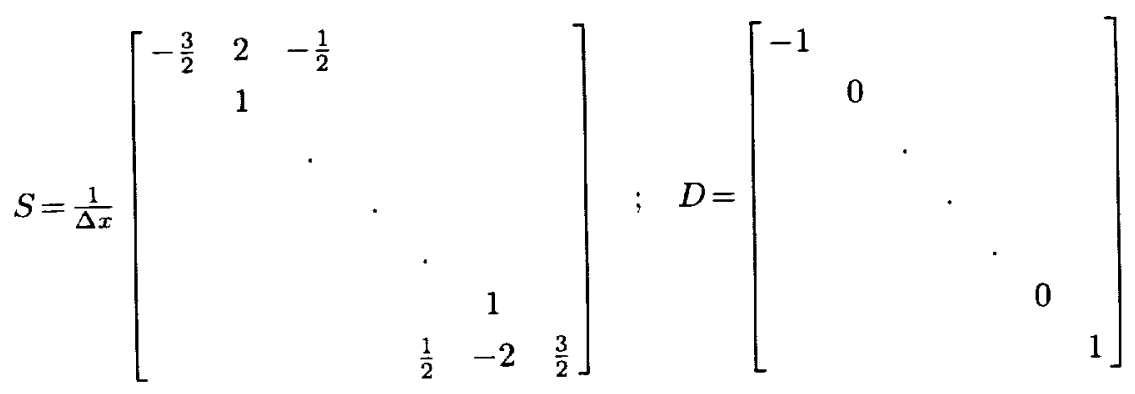

and

$$
R=\frac{1}{(\Delta x)}\left[\begin{array}{cccccccccccc}
\frac{4}{9} & -\frac{2}{9} & \frac{2}{9} & & & & & & & & \\
-\frac{2}{9} & \frac{10}{9} & -\frac{10}{9} & & & & & & & & \\
\frac{2}{9} & -\frac{10}{9} & \frac{19}{9} & -1 & & & & & & & \\
& & -1 & 2 & -1 & & & & & & \\
& & & . & . & . & & & & & & \\
& & & & . & . & . & & & & & \\
& & & & & . & . & . & & & & \\
& & & & & & -1 & 2 & -1 & & \\
& & & & & & & -1 & \frac{19}{9} & -\frac{10}{9} & -\frac{2}{9} \\
& & & & & & & & -\frac{10}{9} & -\frac{10}{9} & \frac{2}{9} \\
& & & & & & & & -\frac{2}{9} & -\frac{2}{9} & \frac{4}{9}
\end{array}\right]
$$

The matrix $R$ can be shown to be positive definite (and symmetric).

The fourth-order discretization that satisfies the SBP constraints was originally derived in the work of Strand [8]. The coefficients $r 1$ and $r 2$ below are different from those proposed by Strand and are chosen so that the resulting discretization $A_{1}=P^{-1} Q$ has the standard four-point third-order stencil at the first grid point. The values of $r 1$ and $r 2$ are

$$
\begin{aligned}
& r 1=\frac{-(2177 \sqrt{295369}-1166427)}{25488} \\
& r 2=\frac{(66195 \sqrt{53} \sqrt{5573}-35909375)}{101952}
\end{aligned}
$$

and the matrices $P$ and $Q$ are

$$
P=\Delta x\left[\begin{array}{cc}
\frac{-(216 r 2+2160 r 1-2125)}{12960} & \frac{(81 r 2+675 r 1+415)}{540} \\
\frac{(81 r 2+675 r 1+415)}{540} & \frac{-(4104 r 2+32400 r 1+11225)}{4320} \\
\frac{-(72 r 2+720 r 1+445)}{1440} & \frac{(1836 r 2+14580 r 1+7295)}{2160} \\
\frac{-(108 r 2+756 r 1+421)}{1296} & \frac{-(216 r 2+2160 r 1+655)}{4320} \\
&
\end{array}\right.
$$
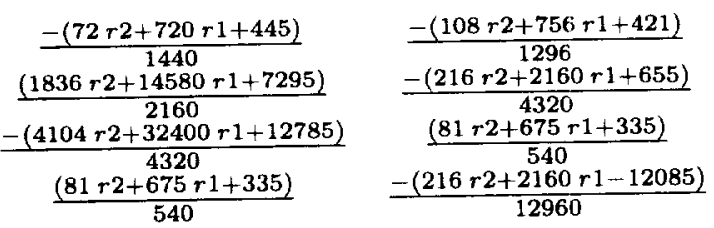
and

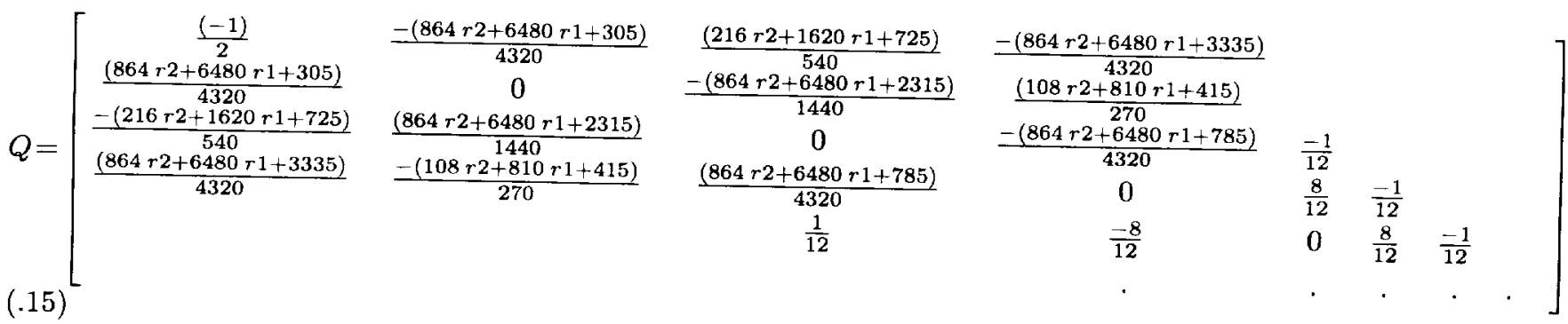

Only the inflow boundary portion of the matrices $P$ and $Q$ is shown. The outflow coefficients are the negative transpose of the inflow coefficients. The matrix $P$ is symmetric and positive definite. is:

The discretization matrix for the diffusion terms that satisfies the constraint $A_{2}=P^{-1}\left(-S^{T} R+D\right) S$

$$
A=\frac{1}{(\Delta x)^{2}}\left[\begin{array}{ccccccccc}
\frac{35}{12} & \frac{-26}{3} & \frac{19}{2} & \frac{-14}{3} & \frac{11}{12} & & & & \\
\frac{11}{12} & \frac{-5}{3} & \frac{1}{2} & \frac{1}{3} & \frac{-1}{12} & & & & \\
\frac{-1}{12} & \frac{16}{12} & \frac{-30}{12} & \frac{16}{12} & \frac{-1}{12} & & & & \\
& \cdot & \cdot & \cdot & \cdot & . & & & \\
& & \cdot & . & . & . & . & & \\
& & & \cdot & . & . & . & . & \\
& & & \frac{-1}{12} & \frac{16}{12} & \frac{-30}{12} & \frac{16}{12} & \frac{-1}{12} \\
& & & \frac{-1}{12} & \frac{1}{3} & \frac{1}{2} & \frac{-5}{3} & \frac{11}{12} \\
& & & \frac{11}{12} & \frac{-14}{3} & \frac{19}{2} & \frac{-26}{3} & \frac{35}{12}
\end{array}\right]
$$

where

$$
S=\frac{1}{\Delta x}\left[\begin{array}{cccccccc}
-\frac{25}{12} & 4 & -3 & \frac{4}{3} & -\frac{1}{4} & & \\
& 1 & & & & & \\
& & \cdot & & & & \\
& & & \cdot & & & \\
& & & & \cdot & & \\
& & & & & 1 & \\
& & -\frac{1}{2} & -\frac{4}{3} & -3 & -4 & \frac{25}{12}
\end{array}\right] ; \quad D=\left[\begin{array}{ccccccc}
-1 & & & & & \\
& 0 & & & & \\
& & & & & \\
& & & & \\
& & & & \\
& & & 0 & \\
& & & & 1
\end{array}\right]
$$

The matrix $R$ is too complicated to report here but can be shown to be positive definite. This numerical scheme is referred to as $(3,3,3,3-4-3,3,3,3)$, which denotes the fact that the four points nearest to the boundary are closed with third-order formulas.

The sixth-order discretization that satisfies the SBP constraints was originally derived in the work of Strand [8]. The coefficients $r 1, r 2$, and $r 3$ below are different from those proposed by Strand and are chosen so that the resulting discretization $A_{1}=P^{-1} Q$ has the standard six-point fifth-order stencil at the first grid point. This choice produces remarkably good stability characteristics at the boundary. The coefficients are

$$
\begin{aligned}
& r 1=-3.6224891259957 \\
& r 2=96.301901955532 \\
& r 3=-609.5813881563
\end{aligned}
$$


The symmetric $P$ and nearly skew-symmetric $Q$ matrices have the entries $A_{1}=P^{-1} Q$, where

$$
\begin{aligned}
& p(1,1)=\frac{-(14400 r 2+302400 r 1-7420003)}{36288000} \\
& p(1,2)=\frac{-(75600 r 3+1497600 r 2+11944800 r 1-59330023)}{21722800} \\
& p(1,3)=\frac{-(9450 r 3+202050 r 2+1776600 r 1-7225847)}{340200} \\
& p(1,4)=\frac{(900 r 2+18900 r 1-649)}{226800} \\
& p(1,5)=\frac{(86400 r 3+1828800 r 2+15854400 r 1-66150023)}{3110400} \\
& p(1,6)=\frac{(378000 r 3+7747200 r 2+65167200 r 1-279318239)}{188640000} \\
& p(2,2)=\frac{(302400 r 3+6091200 r 2+49896000 r 1-210294289)}{7257600} \\
& p(2,3)=\frac{(3780 r 3+82575 r 2+741825 r 1-2991977)}{34020} \\
& p(2,4)=\frac{(5400 r 3+104400 r 2+810000 r 1-3756643)}{129600} \\
& p(2,5)=\frac{-(529200 r 3+11107200 r 2+95508000 r 1-400851749)}{2419200} \\
& p(2,6)=\frac{(86400 r 3+1828800 r 2+15854400 r 1-65966279)}{3110400} \\
& p(3,3)=\frac{-(51300 r 3+1094400 r 2+9585000 r 1-39593423)}{64800} \\
& p(3,4)=\frac{(120960 r 3+2584800 r 2+22680000 r 1-93310367)}{181440} \\
& p(3,5)=\frac{(5400 r 3+104400 r 2+810000 r 1-3766003)}{129600} \\
& p(3,6)=\frac{(900 r 2+18900 r 1-37217)}{226800} \\
& p(4,4)=\frac{-(17100 r 3+364800 r 2+3195000 r 1-13184701)}{21600} \\
& p(4,5)=\frac{(3780 r 3+82575 r 2+741825 r 1-2976857)}{34020} \\
& p(4,6)=\frac{-(1890 r 3+40410 r 2+355320 r 1-1458223)}{68040} \\
& p(5,5)=\frac{(302400 r 3+6091200 r 2+49896000 r 1-213056209)}{7257600} \\
& p(5,6)=\frac{-(75600 r 3+1497600 r 2+11944800 r 1-54185191)}{21722800} \\
& p(6,6)=\frac{-(14400 r 2+302400 r 1-36797603)}{36288000} \\
& q(1,1)=\frac{(-1)}{2} \\
& q(1,2)=\frac{(415800 r 3+8604000 r 2+72954000 r 1-283104553)}{32659200} \\
& q(1,3)=\frac{(120960 r 3+2672640 r 2+24192000 r 1-100358119)}{6531840} \\
& q(1,4)=\frac{-(25200 r 3+542400 r 2+4788000 r 1-19717139)}{403200}
\end{aligned}
$$




$$
\begin{aligned}
& q(1,5)=\frac{(604800 r 3+13363200 r 2+120960000 r 1-485628701)}{32659200} \\
& q(1,6)=\frac{(41580 r 3+860400 r 2+7295400 r 1-31023481)}{3265920} \\
& q(2,2)=0 \\
& q(2,3)=\frac{-(9450000 r 3+200635200 r 2+1747116000 r 1-7286801279)}{32659200} \\
& q(2,4)=\frac{(21168000 r 3+449049600 r 2+3907008000 r 1-16231108387)}{32659200} \\
& q(2,5)=\frac{-(165375 r 3+3516300 r 2+30665250 r 1-126996371)}{453600} \\
& q(2,6)=\frac{(604800 r 3+13363200 r 2+120960000 r 1-482536157)}{32659200} \\
& q(3,3)=0 \\
& q(3,4)=\frac{-(6993000 r 3+148096800 r 2+1286334000 r 1-5353075351)}{8164800} \\
& q(3,5)=\frac{(21168000 r 3+449049600 r 2+3907008000 r 1-16212561187)}{32659200} \\
& q(3,6)=\frac{-(75600 r 3+1627200 r 2+14364000 r 1-58713721)}{1209600} \\
& q(4,4)=0 \\
& q(4,5)=\frac{-(9450000 r 3+200635200 r 2+1747116000 r 1-7263657599)}{32659200} \\
& q(4,6)=\frac{(604800 r 3+13363200 r 2+120960000 r 1-485920643)}{32659200} \\
& q(5,5)=0
\end{aligned}
$$

The matrix $P$ is symmetric and positive definite for this choice of parameters.

The discretization matrix for the diffusion terms that satisfies the constraint $A_{2}=P^{-1}\left(-S^{T} R+D\right) S$ is

$$
A=\frac{1}{180(\Delta x)^{2}}\left[\begin{array}{ccccccc}
+812 & -3132 & +5265 & -5080 & +2970 & -972 & +137 \\
+137 & -147 & -255 & +470 & -285 & +93 & -13 \\
-13 & +228 & -420 & +200 & +15 & -12 & +2 \\
2 & -27 & 270 & -490 & 270 & -27 & 2 \\
& . & . & . & \cdot & . & \cdot
\end{array}\right]
$$

where

$$
S=\frac{1}{\Delta x}\left[\begin{array}{ccccccc}
\frac{(-49)}{20} & 6 & \frac{(-15)}{2} & \frac{20}{3} & \frac{(-15)}{4} & \frac{6}{5} & \frac{(-1)}{6} \\
& 1 & & & & &
\end{array}\right] \quad, \quad D=\left[\begin{array}{cc}
-1 & \\
& 0
\end{array}\right.
$$

The matrix $R$ is too complicated to report here but can be shown to be positive definite. 


\begin{tabular}{|c|c|c|c|}
\hline \multicolumn{3}{|c|}{ REPORT DOCUMENTATION PAGE } & $\begin{array}{l}\text { Form Approved } \\
\text { OMB No. 0704-0188 }\end{array}$ \\
\hline \multicolumn{4}{|c|}{ 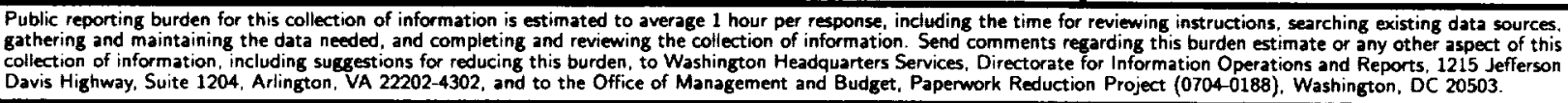 } \\
\hline 1. AGENCY USE ONLY(Leave blank) & $\begin{array}{l}\text { 2. REPORT DATE } \\
\text { February } 1998 \\
\end{array}$ & $\begin{array}{l}\text { 3. REPOR } \\
\text { Contra }\end{array}$ & $\begin{array}{l}\text { VD DATES COVERED } \\
\text { ort }\end{array}$ \\
\hline \multicolumn{3}{|c|}{$\begin{array}{l}\text { 4. TITLE AND SUBTITLE } \\
\text { A Stable and Conservative Interface Treatment of Arbitrary Spatial } \\
\text { Accuracy }\end{array}$} & $\begin{array}{l}\text { 5. FUNDING NUMBERS } \\
\text { C NAS1-19480 } \\
\text { C NAS1-97046 }\end{array}$ \\
\hline \multicolumn{3}{|l|}{$\begin{array}{l}\text { 6. AUTHOR(5) } \\
\text { Mark H. Carpenter } \\
\text { Jan Nordstrom } \\
\text { David Gottlieb }\end{array}$} & WU 505-90-52-01 \\
\hline \multicolumn{3}{|c|}{$\begin{array}{l}\text { 7. PERFORMING ORGANIZATION NAME(S) AND ADDRESS(ES) } \\
\text { Institute for Computer Applications in Science and Engineering } \\
\text { Mail Stop 403, NASA Langley Research Center } \\
\text { Hampton, VA 23681-2199 }\end{array}$} & $\begin{array}{l}\text { 8. PERFORMING ORGANIZATION } \\
\text { REPORT NUMBER } \\
\text { ICASE Report No. } 98-12\end{array}$ \\
\hline \multicolumn{3}{|c|}{$\begin{array}{l}\text { 9. SPONSORING/MONITORING AGENCY NAME(S) AND ADDRESS(ES) } \\
\text { National Aeronautics and Space Administration } \\
\text { Langley Research Center } \\
\text { Hampton, VA 23681-2199 }\end{array}$} & $\begin{array}{l}\text { 10. SPONSORING/MONITORING } \\
\text { AGENCY REPORT NUMBER } \\
\text { NASA/CR-1998-206921 } \\
\text { ICASE Report No. } 98-12\end{array}$ \\
\hline
\end{tabular}

Langley Technical Monitor: Dennis M. Bushnell

Final Report

Submitted to Journal of Computational Physics

12a. DISTRIBUTION/AVAILABILITY STATEMENT

12b. DISTRIBUTION CODE

Unclassified Unlimited

Subject Category 64

Distribution: Nonstandard

Availability: NASA-CASI (301)621-0390

13. ABSTRACT (Maximum 200 mords)

Stable and accurate interface conditions are derived for the linear advection-diffusion cquation. The conditions are functionally independent of the spatial order of accuracy and rely only on the form of the discrete operator. We focus on high-order finite-difference operators that satisfy the summation-by-parts (SBP) property. We prove that stability is a natural consequence of the SBP operators used in conjunction with the new boundary conditions. In addition, we show that the interface treatments are conservative.

New finite-difference operators of spatial accuracy up to sixth order are constructed: these operators satisfy the SBP property. Finite-difference operators are shown to admit design accuracy ( $p^{t h}$-order global accuracy) when $(p-1)^{\text {th }}$-order stencil closures are used near the boundaries if the physical boundary conditions are implemented to at least $p^{\text {th }}$-order accuracy. Stability and accuracy are demonstrated on the nonlinear Burgers' equation for an twelve-subdomain problem with randomly distributed interfaces.

\section{SUBJECT TERMS}

high-order finite-difference, numerical stability, interface conditions, summation-byparts

17. SECURITY CLASSIFICATION
OF REPORT
Unclassified

18. SECURITY CLASSIFICATION
OF THIS PAGE
Unclassified

19. SECURITY CLASSIFICATION OF ABSTRACT

15. NUMBER OF PAGES 24

16. PRICE CODE $\mathrm{A} 03$

20. LIMITATION OF ABSTRACT 